\title{
Revisiting Digital Straight Segment Recognition
}

\author{
François de Vieilleville and Jacques-Olivier Lachaud \\ LaBRI, Univ. Bordeaux 1 \\ 351 cours de la Libération, \\ 33405 Talence Cedex, France \\ $\{$ devieill, lachaud\}@labri.fr
}

\begin{abstract}
This paper presents new results about digital straight segments, their recognition and related properties. They come from the study of the arithmetically based recognition algorithm proposed by I. Debled-Rennesson and J.-P. Reveillès in 1995 1]. We indeed exhibit the relations describing the possible changes in the parameters of the digital straight segment under investigation. This description is achieved by considering new parameters on digital segments: instead of their arithmetic description, we examine the parameters related to their combinatoric description. As a result we have a better understanding of their evolution during recognition and analytical formulas to compute them. We also show how this evolution can be projected onto the Stern-Brocot tree. These new relations have interesting consequences on the geometry of digital curves. We show how they can for instance be used to bound the slope difference between consecutive maximal segments.
\end{abstract}

\section{Introduction}

The study of digital straight lines is a fundamental topic in discrete geometry and several approaches have been proposed. Following the taxonomy of [2, we can divide them into three groups. The first one characterizes digital lines through the study of the pre-image: this aims at determining in a dual space the possible real lines whose digitization corresponds to a given set of pixels 3 . That kind of approaches has recently been used to define and recognize straight lines drawn on irregular isothetic grids 4].

A second group is related to combinatorics and relies on the link between continued fractions and recursive characterization of digital lines. It can cope with lines with rational or irrational slopes, as their digitization can be seen as a word over a finite alphabet. The tools developed to characterize and study those objects [5, 6, have for instance been used to study the asymptotic behavior of some digital segments over digitizations of $\mathcal{C}^{3}$ convex curves [7, 8].

The third group gathers arithmetic approaches, which are based on a formulation very similar to the one of real lines (Diophantine inequalities, slope and vertical shift). They have led to simple, incremental and optimal algorithms to recognize digital segments [1,9]. For this approach the best known recognition algorithm is the above mentioned algorithm of Debled and Reveillès [1, referenced as DR95 algorithm in the recent book of Klette and Rosenfeld [10]. This 
algorithm extracts progressively the most simple digital line parameters of a finite connected sequence of pixels, updating the parameters at each pixel.

In this algorithm, the parameters refer to an arithmetic representation of digital straight lines: slope as a fraction, integer shift to origin, position of some specific limit points (upper and lower leaning points). Their evolution during the progressive steps of the recognition is governed by algorithmic computations: for instance, the new slope is computed from the last added point and some former leaning point. Although sufficient for recognizing digital lines, these parameters lack of descriptive content to fully understand what is digital straightness. For instance, they cannot answer a question like if two straight lines share a common part, how are related their slopes. Along the same lines, although it is known since Debled's thesis 11 that slope evolutions during recognition correspond to displacements in the Stern-Brocot tree, these parameters are nevertheless incomplete to fully describe it.

We propose here to use the combinatoric approach to give better insights about the DR95 algorithm. A digital line is then characterized by the continued fraction of its slope and the number of patterns it contains. The evolution of these new parameters is then precisely stated with analytic formulas. We also give another interpretation of their evolution, as definite displacements on the Stern-Brocot tree. Afterwards we focus on a particular class of digital segments subset of digital curves, which are called maximal segments and which have interesting properties [12,13, 14. Informally, they form the inextensible digital straight segments on the curve. The preceding properties allow us to give an analytic writing of the minimal and maximal slope variation between two consecutive maximal segments. These bounds are fully described with our new parameters. Surprisingly, they show that consecutive maximal segments may not vary too much nor too little since both bounds are of the same order wrt parameters. On a long term, these quantitative relations will be crucial for designing digital curvature estimators based on slope variations.

This paper is organized as follows. First, basic arithmetic and combinatoric definitions and properties of digital lines are recalled (Section 2). Then we give a comprehensive explanation of the DR95 algorithm, describing each possible evolution in terms of the new parameters (Section 3). Afterwards the connection between the DR95 and the Stern-Brocot tree is explicited (Section 4). Eventually those properties have consequences on the geometry of maximal segments, namely bounds on their slope variations (Section 5). We conclude the paper by some perspectives to this work (Section 6).

\section{Digital Straight Segments: Arithmetic and Combinatoric Approach}

Given a compact set with rectifiable boundary we consider its Gauss digitization. The digital border of this digitization is chosen as the inter-pixel 4-connected path laying between its inner and outer digitization. This digital curve is referred as $C$. We consider that the points on the boundary are indexed increasingly, 
for instance with a counterclockwise order. Moreover, given two points on the boundary (say $A$ and $B$ ), $C_{A, B}$ is the digital path from $A$ to $B$. For convenience reasons we identify the index of a point on the boundary to the point itself. For example $A<B$ means that the point $A$ is before the point $B$ on the curve.

Let us recall the arithmetic definition of digital straight lines and explain the notations that will be used in the following of the paper. Following definitions hold in the first octant.

Definition 1. The set of points $(x, y)$ of the digital plane verifying $\mu \leq a x-b y<$ $\mu+|a|+|b|$, with $a, b$ and $\mu$ integer numbers, is called the standard line with slope $a / b$ and shift $\mu$ [15] (e.g. see Fig. 11).

The standard lines are the 4-connected discrete lines. The quantity $r_{(a, b)}(P)=$ $a x-b y$ is the remainder of the point $P=(x, y)$ in the digital line of characteristics $(a, b, \mu)$. The points whose remainder is $\mu$ (resp. $\mu+|a|+|b|-1)$ are called upper (resp. lower) leaning points.

Definition 2. A set of successive points $C_{i, j}$ of the digital curve $C$ is a digital straight segment (DSS) iff there exists a standard line $(a, b, \mu)$ containing them. The predicate " $C_{i, j}$ is a DSS" is denoted by $S(i, j)$. When $S(i, j)$ the characteristics associated with the DSS $C_{i, j}$ (extracted with the DR95 algorithm) [1] are the characteristics $(a, b, \mu)$ which minimize $a+b$.

The original DR95 algorithm recognizes naive digital straight line but it is easily adapted to standard lines. It extracts the characteristics $(a, b, \mu)$, with minimal $a+b$. The evolution of the characteristics is based on a simple test: each time we try to add a new point 4-connected to the current digital straight segment, we compute its remainder with respect to the DSS parameters. According to this value the point can be added or not. If it is greater than or equal to $\mu+a+b+1$ or less than or equal to $\mu-2$ the point is said to be exterior to the digital straight segment and cannot be added. Otherwise the point can be added to the segment to form a longer DSS and falls into two categories:

- interior points, with a remainder between $\mu$ and $\mu+a+b-1$ both included;

- weakly exterior points, with a remainder of $\mu-1$ for upper weakly exterior points and $\mu+a+b$ for lower weakly exterior points. Only in this case are the characteristics updated.

Even if the arithmetic approach is a powerful tool for digital straight segment recognition, other approaches may reveal useful to get analytic properties. We here recall one of those approaches which is connected to continued fractions.

Definition 3. Given a standard line of characteristics $(a, b, \mu)$, we call pattern of characteristics $(a, b)$ the word formed by the Freeman codes between any two consecutive upper leaning points. The Freeman codes defined between any two consecutive lower leaning points is the previous word read from back to front and is called the reversed pattern of characteristics $(a, b)$.

Since a DSS has at least either two upper or two lower leaning points, a DSS $(a, b, \mu)$ contains at least one pattern or one reversed pattern of characteristics 


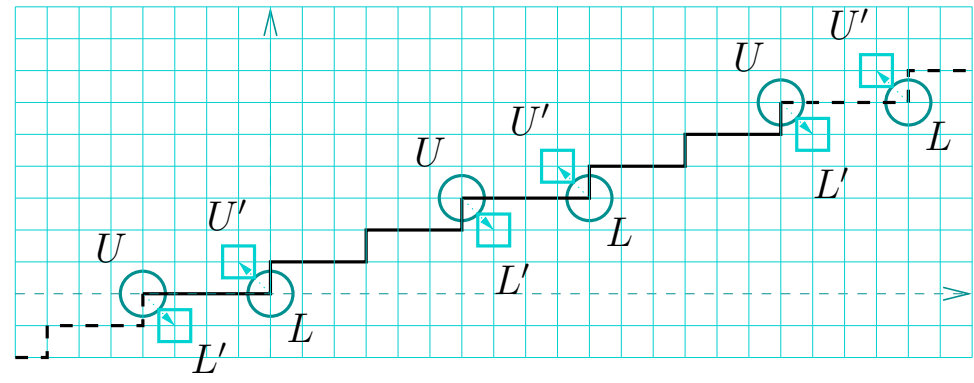

Fig. 1. Positions of weakly exterior points on a digital straight line of characteristics $(3,10,12)$. Weakly exterior points are boxed and leaning points are circled.

$(a, b)$. It is important to note that a DSS $(a, b, \mu)$ contains $\delta$ pattern $(a, b)$ (resp. $\delta^{\prime}$ reversed-pattern $\left.(a, b)\right)$ iff it has $\delta+1$ upper leaning points (resp. $\delta^{\prime}+1$ lower leaning points). Moreover for any $\operatorname{DSS}(a, b, \mu)$, the number of pattern $(a, b)$ and reversed-pattern $(a, b)$ differ from one.

There exists recursive transformations for computing the pattern of a standard line from the simple continued fraction of its slope ( [5], [10] Chap. 9 or [6] Chap. 4). We chose to focus on Berstel's approach, which better suits our purpose. A continued fraction $z$ will be conveniently denoted by $\left[0, u_{1} \ldots, u_{n}, \ldots\right]$. The $u_{i}$ are called elements or partial coefficients and the continued fraction formed with the $k+1$ first partial coefficients of $z$ is said to be a $k$-th convergent of $z$ and is denoted $z_{k}$. The depth of a $k$-th convergent equals $k$. We conveniently denote by $p_{k}$ the numerator and by $q_{k}$ the denominator of a $k$-th convergent.

We recall a few more relations regarding the way convergents can be formed:

$$
\begin{array}{llll} 
& & \forall k \geq 1 & p_{k} q_{k-1}-p_{k-1} q_{k}=(-1)^{k+1}, \\
p_{0}=0 & p_{-1}=1 & \forall k \geq 1 & p_{k}=u_{k} p_{k-1}+p_{k-2}, \\
q_{0}=1 & q_{-1}=0 & \forall k \geq 1 & q_{k}=u_{k} q_{k-1}+q_{k-2} .
\end{array}
$$

Continued fractions can be finite or infinite, we focus on the case of rational slopes of lines in the first octant, that is finite continued fractions between 0 and 1. For each $i, u_{i}$ is assumed to be a strictly positive integer. In order to have a unique writing we consider that the last partial coefficient is greater or equal to two; except for slope $1=[0,1]$.

Let us now explain how to compute the pattern associated with a rational slope $z$ in the first octant (i.e. $z=\frac{a}{b}$ with $0 \leq a<b$ ). Horizontal steps are denoted by 0 and vertical steps are denoted by 1 . Let us define $E$ a mapping from the set of positive rational number smaller than one onto the Freemancode's words, more precisely $E\left(z_{0}\right)=0, E\left(z_{1}\right)=0^{u_{1}} 1$ and others values are expressed recursively:

$$
\begin{aligned}
E\left(z_{2 i+1}\right) & =E\left(z_{2 i}\right)^{u_{2 i+1}} E\left(z_{2 i-1}\right), \\
E\left(z_{2 i}\right) & =E\left(z_{2 i-2}\right) E\left(z_{2 i-1}\right)^{u_{2 i}} .
\end{aligned}
$$




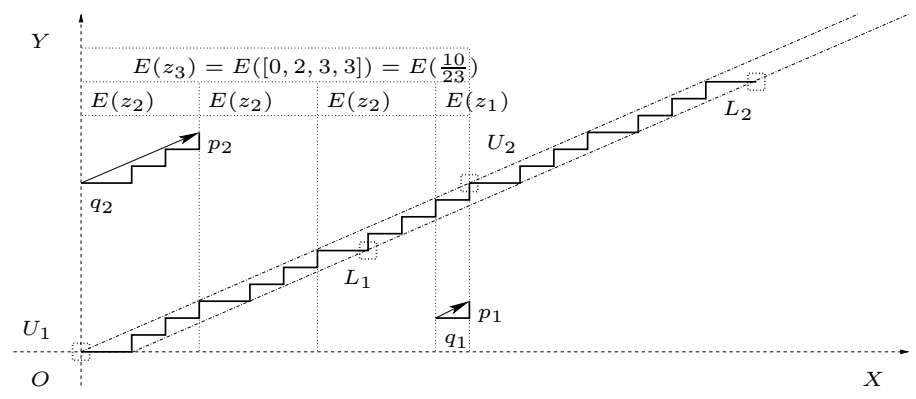

Fig. 2. A digital straight segment of characteristics $(10,23,0)$ with an odd slope, taken between origin and its second lower leaning point

It has been shown that this mapping constructs the pattern $(a, b)$ for any rational slope $z=\frac{a}{b}$. Fig. 2 exemplifies the construction.

There exists other equivalent relations for computing numerators and denominators (see [10] Chap. 9 and [6] Chap. 4) and the splitting formula can be used to obtain patterns. However the splitting formula uses two $k$-th convergent with the same depth, whereas we here use two $k$-th convergent of consecutive depth. The parity of a slope is defined as the parity of the depth of its development in continued fractions.

\section{Combinatoric View of DR95 Algorithm}

Changes in the slope with the DR95 algorithm occur when weakly exterior points are added to the segment. We propose here to explain the different classes of parameters that rule the evolution process, that is, the characteristics of the straight segment $(a, b, \mu)$, the numbers of patterns and reversed-pattern $(a, b)$ that constitute it, the depth of the rational fraction $\frac{a}{b}$, the type of weakly exterior point that is added (upper or lower) and if it is added to the front or to the back.

If a digital straight segment with characteristics $(a, b, \mu)$ does not contain any pattern $(a, b)$ then it only contains a reversed-pattern $(a, b)$, that is, two lower leaning points and one upper leaning point.

Lemma 1. If a digital straight segment does not contain any pattern, then there is necessarily one upper leaning point laying on the digital path before an upper weakly exterior point. Similarly, if a digital straight segment does not contain any reversed-pattern, then there is necessarily one lower leaning point laying on the digital path before a lower weakly exterior point.

Proof. Due to the values of the remainder, one can see that weakly exterior points and leaning points are connected (Fig. 11). Consider a digital straight line of characteristics $(a, b, \mu)$, and let $U$ (resp. $L$ ) be an upper (resp. lower) leaning point of that line. The point $U+(1,-1)$ has a remainder of $\mu+a+b$ which means it is a lower weakly exterior point. A similar reasoning shows us 
that $L+(-1,1)$ is always an upper weakly exterior point. As a result, leaning points and weakly exterior points are ordered in a particular way. We can therefore state that before a weakly exterior point lays a leaning point of the same type.

Thus we consider that before adding an upper weakly exterior point to a digital straight segment, $\delta$ is always greater than or equal to one. Similarly before adding a lower weakly exterior point to a digital segment, $\delta^{\prime}$ is always greater than or equal to one. Let us state precisely the evolution of the slope of a segment when adding an upper weakly exterior point to its back or its front.

Proposition 1. The evolution of the slope of a DSS recognized with the DR95 algorithm depends of the parity of its depth, the type of weakly exterior point added and the side where it is added. This process can be summed up as follows:

- slope with even depth $\left[0, u_{1}, \ldots, u_{2 i}\right], \delta$ pattern $(s)$ and $\delta^{\prime}$ reversed-pattern $(s)$ :

\begin{tabular}{|l|c|c|}
\cline { 2 - 3 } \multicolumn{1}{c|}{} & Back side & Front side \\
\hline Upper weakly exterior & {$\left[0, u_{1}, \ldots, u_{2 i}-1,1, \delta\right]$} & {$\left[0, u_{1}, \ldots, u_{2 i}, \delta\right]$} \\
\hline Lower weakly exterior & {$\left[0, u_{1}, \ldots, u_{2 i}, \delta^{\prime}\right]$} & {$\left[0, u_{1}, \ldots, u_{2 i}-1,1, \delta^{\prime}\right]$} \\
\hline
\end{tabular}

- slope with odd depth $\left[0, u_{1}, \ldots, u_{2 i+1}\right], \delta$ pattern $(s)$ and $\delta^{\prime}$ reversed-pattern $(s)$ :

\begin{tabular}{|c|c|c|}
\cline { 2 - 3 } \multicolumn{1}{c|}{} & Back side & Front side \\
\hline Upper weakly exterior & {$\left[0, u_{1}, \ldots, u_{2 i+1}, \delta\right]$} & {$\left[0, u_{1}, \ldots, u_{2 i+1}-1,1, \delta\right]$} \\
\hline Lower weakly exterior & {$\left[0, u_{1}, \ldots, u_{2 i+1}-1,1, \delta^{\prime}\right]$} & {$\left[0, u_{1}, \ldots, u_{2 i+1}, \delta^{\prime}\right]$} \\
\hline
\end{tabular}

Proof. We give the proof in the case of an even slope when an upper weakly exterior point is added to the right side. Other cases are deduced with a similar reasoning or considering the segment upside-down.

Consider we have $\delta \geq 1$. Let $U_{L}$ and $U_{R}$ be the leftmost and rightmost upper leaning point of the DSS. We choose $U_{L}$ as the origin. Let $\frac{p_{2 i}}{q_{2 i}}=\left[0 ; u_{1}, \ldots, u_{2 i}\right]$.

The added point $U^{\prime}$ has a remainder equal to -1 . Eq. (11) indicates that $q_{2 i-1}$ and $p_{2 i-1}$ are the smallest positive Bezout coefficient verifying $p_{2 i} x-q_{2 i} y=-1$. As a result: $\mathbf{U}_{\mathbf{R}} \mathbf{U}^{\prime}=\left(q_{2 i-1}, p_{2 i-1}\right)$.

From the recognition algorithm DR95 the slope of the segment $C_{U_{L}, U^{\prime}}$ is given by the vector $\mathbf{U}_{\mathbf{L}} \mathbf{U}^{\prime}$. Since $\mathbf{U}_{\mathbf{L}} \mathbf{U}^{\prime}=\mathbf{U}_{\mathbf{L}} \mathbf{U}_{\mathbf{R}}+\mathbf{U}_{\mathbf{R}} \mathbf{U}^{\prime}, \mathbf{U}_{\mathbf{L}} \mathbf{U}^{\prime}$ equals $\delta\left(q_{2 i}, p_{2 i}\right)+$ $\left(q_{2 i-1}, p_{2 i-1}\right)$. From Eq. (2) and (3) this slope equal $\frac{p_{2 i+1}}{q_{2 i+1}}=$ $\left[0, u_{1}, \ldots, u_{2 i}, \delta\right]$. If $\delta$ equals one then $\left[0, u_{1}, \ldots, u_{2 i}, 1\right]=\left[0, u_{1}, \ldots, u_{2 i}+1\right]$.

The slope depth of a DSS when adding a weakly exterior point remains the same or is increased by one or two.

\section{Connection with the Stern-Brocot Tree}

We now show that the evolution of the DSS parameters during the recognition process, analytically given in Proposition 1, can be traced on the Stern-Brocot tree and has then a more intuitive interpretation. The Stern-Brocot tree represents all positive rational fractions. It was already observed that the DR95 
recognition algorithm has some connection with it [11]. More precisely the successive values of the slope taken by a segment are deeper and deeper nodes of this tree. Note that this tree has other connections with discrete geometry, like determining the minimal characteristics of the intersection of two digital straight lines 16, 17.

The Stern-Brocot tree is a binary tree constructed by starting with the fractions $\frac{0}{1}$ and $\frac{1}{0}$ and iteratively inserting $\frac{m+m^{\prime}}{n+n^{\prime}}$ between each two adjacent fractions $\frac{m}{n}$ and $\frac{m^{\prime}}{n^{\prime}}$ (Fig. 33). Any node with a value between 0 (excluded) and 1 (included) is obtained by finite successive moves from the $\frac{1}{1}$ node. Those moves can be of two types: $L$ is a move toward the left child, $R$ is a move toward the right child. Those moves determine the type of node when they end paths: even (resp. odd) nodes end with a $R$ (resp. $L$ ) move. It is known that those nodes have a development in continued fraction, and is such that:

- even nodes:

$\left[0, u_{1}, \ldots, u_{2 k}, u_{2 k+1}, \ldots, u_{2 i-1}, u_{2 i}\right] \equiv R^{0} L^{u_{1}} \ldots R^{u_{2 k}} L^{u_{2 k+1}} \ldots L^{u_{2 i-1}} R^{u_{2 i}-1}$

- odd nodes:

$\left[0, u_{1}, \ldots, u_{2 k}, u_{2 k+1}, \ldots, u_{2 i}, u_{2 i+1}\right] \equiv R^{0} L^{u_{1}} \ldots R^{u_{2 k}} L^{u_{2 k+1}} \ldots R^{u_{2 i}} L^{u_{2 i+1}-1}$

Of course odd nodes have an odd depth in their development in continued fractions, similarly even nodes have an even depth. When descending the tree the depth of a child changes if the move used to reach it differs from the last move used to reach its father. Consider the node $\frac{1}{2}$ whose depth equals one, the last move used to reach it is a $L$ move, its left child $\frac{1}{3}$ has the same depth. The right child of $\frac{1}{2}$ is obtained by the successive moves $R^{0} L^{1} R^{1}$ and has a depth that equals two. We can classify the nodes of the Stern-Brocot tree according to the depth of their development in continued fraction (Fig. 33).

Nodes of this tree may also be viewed as slopes of a digital straight segment being recognized. As we consider rational slopes between zero and one, we only consider nodes whose value is between zero and one. All those nodes are derived (except for the zero node) from the $\frac{1}{1}$ node with a $L$ move first. This implies that $u_{0}$ equals zero. It is possible to trace the slope evolution of a digital straight segment during recognition on the Stern-Brocot tree, as exemplified in Fig. 3.

Since each node has a particular development in continued fraction, the results shown in Proposition 1 can be reinterpreted in terms of descending moves on the Stern-Brocot tree. Thus the slope evolution of a DSS during recognition can be fully described with $L$ and $R$ moves as shown on Fig. 4 , We then see that the number of successive moves of the same type directly depends on the number of patterns or reversed-patterns.

From Fig. 4 we can see that the left child nodes are always reached when we add to the right a lower weakly exterior point or to the left an upper weakly exterior point whatever the parity of the node depth. Same reasoning applies for right child nodes. We can now see how the slope evolution is translated as moves on the Stern-Brocot tree when adding a point. Fig. 5 pictures the possible slope evolutions from the $\frac{1}{2}$ node. 


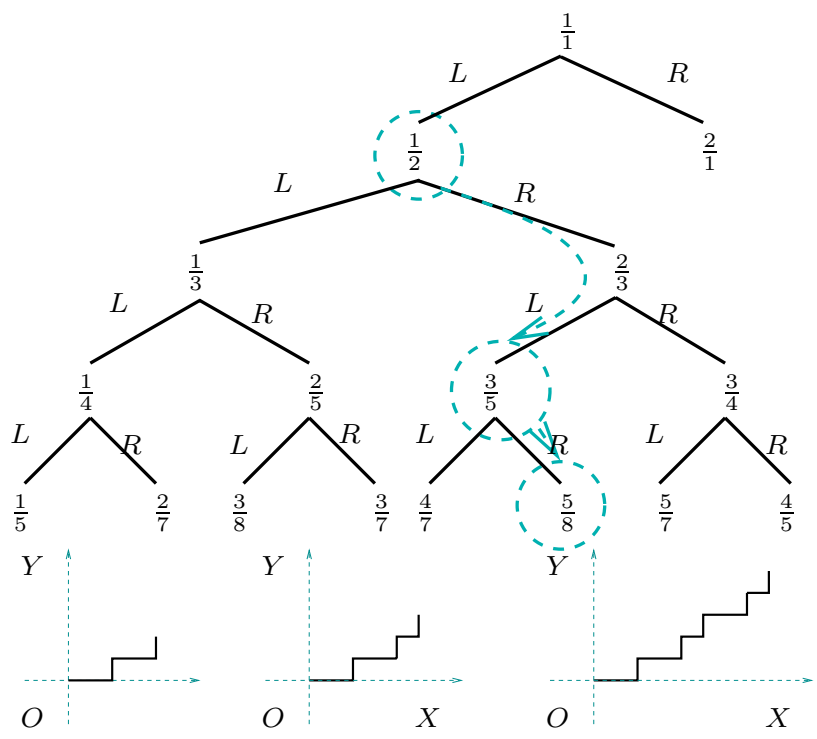

Fig. 3. Evolution of the slope of a digital straight segment. Successive values are $\frac{1}{2}$, $\frac{3}{5}$ and $\frac{5}{8}$. Modifications are triggered by the addition of an upper weakly exterior point. The successive slope depths are one, three and four. Nodes with depth one are $\frac{1}{1}, \frac{1}{2}, \frac{1}{3}, \frac{1}{4}, \frac{1}{5}$, nodes with depth two are $\frac{2}{3}, \frac{3}{4}, \frac{4}{5}, \frac{2}{5}, \frac{3}{7}, \frac{2}{7}$ nodes with depth three are $\frac{3}{5}, \frac{4}{7}, \frac{3}{8}, \frac{5}{7}$ and node with depth four is $\frac{5}{8}$.

\section{Application to Maximal Segments}

We now apply previous properties to get a better understanding of the geometry of maximal segments. Maximal segments form a particular class of digital straight segments on the digital curve. Their study is related to many discrete geometry problems such as digital convexity [14, polygonalization [12] or tangent computation 13. The main result of this section is Theorem 1 which bounds the slope difference between two consecutive maximal segments. Let us first explain how to characterize them.

Given a point $C_{i}$ on a digital curve $C$, the first index $j$ greater than $i$ such that $S(i, j)$ and $\neg S(i, j+1)$ is called the front of $i$. The map associating any $i$ to its front is denoted by $F$. Symmetrically, the first index $i$ such that $S(i, j)$ and $\neg S(i-1, j)$ is called the back of $j$ and the associated mapping is denoted by $B$.

Definition 4. Any set of points $C_{i, j}$ is called a maximal segment iff any of the following equivalent characterizations holds: (1) $S(i, j)$ and $\neg S(i, j+1)$ and $\neg S(i-1, j)$, (2) $B(j)=i$ and $F(i)=j$.

Maximal segments form the set of DSS on the digital curve that cannot be extended on any side. They can be ordered along the curve. Consecutive maximal segments overlap and often on more than two points. The digital path that belongs to two consecutive maximal segments is called a common part, its as- 


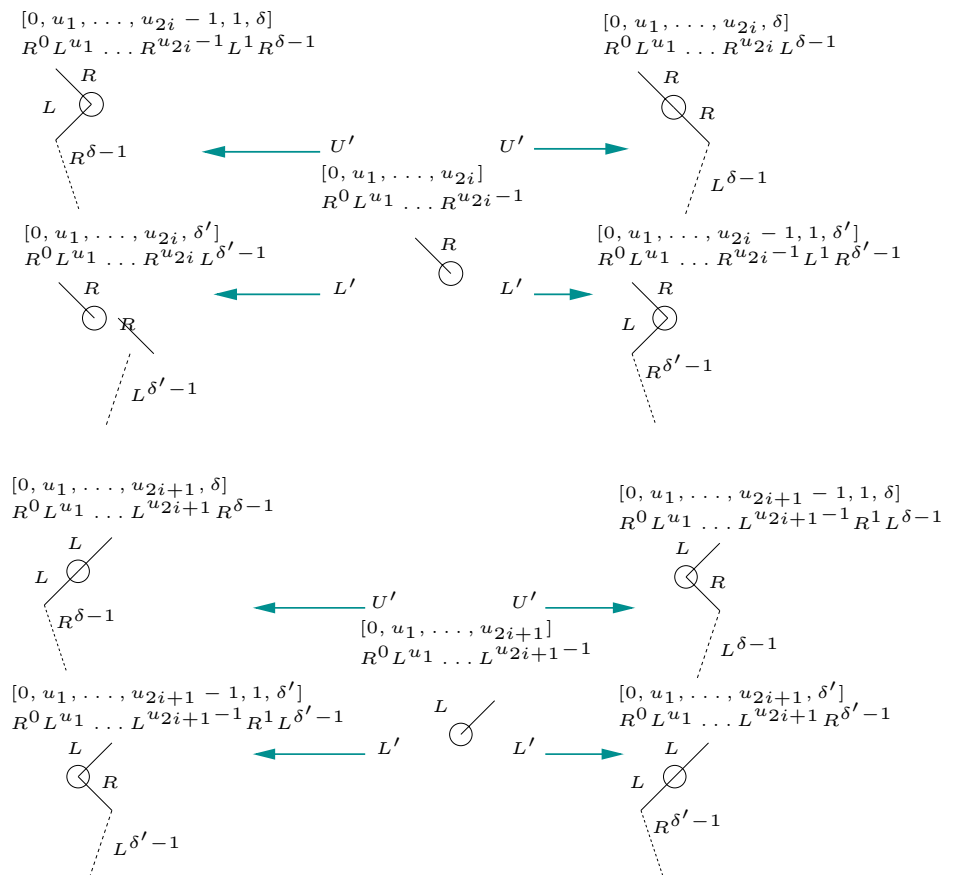

Fig. 4. Evolution from a digital straight segment with even slope (top) and odd slope (bottom) using the DR95 algorithm, represented in terms of move on the Stern-Brocot tree. The point $U^{\prime}$ (resp. $L^{\prime}$ ) is an upper (resp. lower) weakly exterior point added to the back (left column) or to the front (right column) of the DSS.

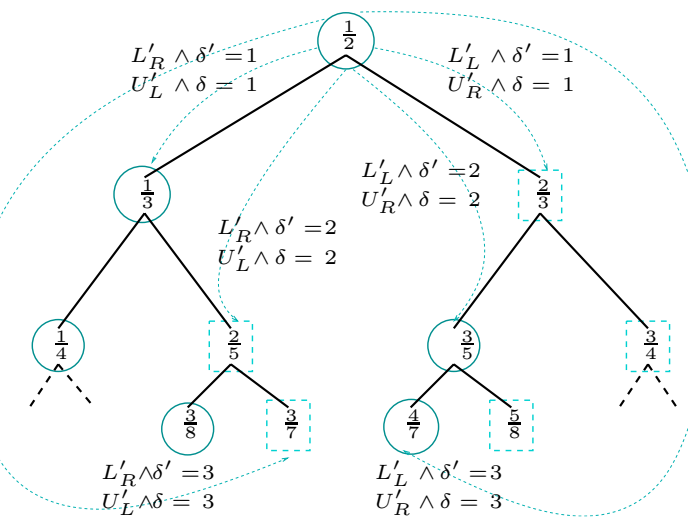

Fig. 5. Reachable slopes when a weakly exterior point is added to a digital straight segment of slope $\frac{1}{2}$. Nodes with an odd depth are circled, while those with even depth are dash-boxed. $L_{L}^{\prime}$ and $U_{L}^{\prime}$ (resp. $L_{R}^{\prime}$ and $U_{R}^{\prime}$ ) stand for lower and upper weakly exterior point added to the back (resp. front). $\delta$ and $\delta^{\prime}$ count the number of patterns and reversed-patterns respectively. 
sociated maximal segments are $C_{B(j), j}$ and $C_{i, F(i)}$ if $C_{i, j}$ is the common part. Note that $C_{i-1, j+1}$ is not a DSS. A common part is never empty (though it may be reduced to two points). In fact we know the type of the limiting points for all common parts, as shown below:

Lemma 2. If $C_{i, j}$ is the common part of two consecutive maximal segments, then the points $i-1$ and $j+1$ are both upper or both lower weakly exterior.

Proof. $C_{i, F(i)}$ is one of the two consecutive maximal segments, thus $F(i)>j$ and $S(i, j+1)$ holds. The point $j+1$ is thus an interior point or a weakly exterior point for $C_{i, j}$. Assuming that $j+1$ is an interior point for $C_{i, j}$, any extensions to the back of $C_{i, j}$ is compatible with $C_{i, j+1}$. The maximal segment $C_{B(j), j}$ is one of these extensions, thus $S(B(j), j+1)$ would hold which raises a contradiction. As a consequence $j+1$ is a weakly exterior point for the DSS $C_{i, j}$ and a similar reasoning can be applied to $i-1$.

We prove by contradiction that $i-1$ and $j+1$ are either both lower or both upper weakly exterior. Assume $i-1$ is upper weakly exterior and $j+1$ is lower weakly exterior. Let the DSS $C_{i, j}$ be constituted of $\delta$ patterns and $\delta^{\prime}$ reversedpatterns. By definition, $\delta$ and $\delta^{\prime}$ differ at most of one but here, given the type of $i-1$ and $j+1$, the equality $\delta=\delta^{\prime}$ holds. From Proposition 1, DSS $C_{i-1, j}$ and $C_{i, j+1}$ have the same slope (whichever the parity). Furthermore the DR95 algorithm for updating slopes indicates they share the same leaning points $L_{L}$ and $U_{R}$. These two assertions, combined together, entail $C_{i-1, j+1}$ is a DSS too, which contradicts the hypothesis that it is a common part.

We give now analytic bounds on slopes of two consecutive maximal segments.

Theorem 1. If $C_{i, j}$ is the common part of two consecutive maximal segments (namely $C_{B(j), j}$ and $\left.C_{i, F(i)}\right)$, their slopes are such that:

\begin{tabular}{|l|c|c|c|c|c|}
\cline { 3 - 6 } \multicolumn{2}{c|}{} & \multicolumn{4}{|c|}{$i-1$ and $j+1$ are both (Lemma 2) } \\
\cline { 3 - 6 } \multicolumn{2}{c|}{} & \multicolumn{2}{c|}{ lower weakly exterior } & \multicolumn{2}{c|}{ upper weakly exterior } \\
\cline { 3 - 7 } & minimal slope & maximal slope & minimal slope & maximal slope \\
\hline \multirow{2}{*}{$\begin{array}{l}C_{i, j} \text { has an } \\
\text { even slope }\end{array}$} & $C_{i, F(i)}$ & $\frac{\delta^{\prime} p_{2 i}-p_{2 i-1}}{\delta^{\prime} q_{2 i}-q_{2 i-1}}$ & $\frac{\left.\delta^{\prime}+2\right) p_{2 i}-p_{2 i-1}}{\left(\delta^{\prime}+2\right) q_{2 i}-q_{2 i-1}}$ & $\frac{(\delta+1) p_{2 i}+p_{2 i-1}}{(\delta+1) q_{2 i}+q_{2 i-1}}$ & $\frac{(\delta-1) p_{2 i}+p_{2 i-1}}{(\delta-1) q_{2 i}+q_{2 i-1}}$ \\
\cline { 2 - 7 } & $C_{B(j), j}$ & $\frac{\left(\delta^{\prime}+1\right) p_{2 i}+p_{2 i-1}}{\left(\delta^{\prime}+1\right) q_{2 i}+q_{2 i-1}}$ & $\frac{\left(\delta^{\prime}-1\right) p_{2 i}+p_{2 i-1}}{\left(\delta^{\prime}-1\right) q_{2 i}+q_{2 i-1}}$ & $\frac{\delta p_{2 i}-p_{2 i-1}}{\delta q_{2 i}-q_{2 i-1}}$ & $\frac{(\delta+2) p_{2 i}-p_{2 i-1}}{(\delta+2) q_{2 i}-q_{2 i-1}}$ \\
\hline \multirow{2}{*}{$\begin{array}{l}C_{i, j} \text { has an } \\
\text { odd slope }\end{array}$} & $C_{i, F(i)}$ & $\frac{\left(\delta^{\prime}+1\right) p_{2 i+1}+p_{2 i}}{\left(\delta^{\prime}+1\right) q_{2 i+1}+q_{2 i}}$ & $\frac{\left(\delta^{\prime}-1\right) p_{2 i+1}+p_{2 i}}{\left(\delta^{\prime}-1\right) q_{2 i+1}+q_{2 i}}$ & $\frac{\delta p_{2 i+1}-p_{2 i}}{\delta q_{2 i+1}-q_{2 i}}$ & $\frac{(\delta+2) p_{2 i+1}-p_{2 i}}{(\delta+2) q_{2 i+1}-q_{2 i}}$ \\
\cline { 2 - 7 } & $C_{B(j), j}$ & $\frac{\delta^{\prime} p_{2 i+1}-p_{2 i}}{\delta^{\prime} q_{2 i+1}-q_{2 i}}$ & $\frac{\left(\delta^{\prime}+2\right) p_{2 i+1}-p_{2 i}}{\left(\delta^{\prime}+2\right) q_{2 i+1}-q_{2 i}}$ & $\frac{(\delta+1) p_{2 i+1}+p_{2 i}}{(\delta+1) q_{2 i+1}+q_{2 i}}$ & $\frac{(\delta-1) p_{2 i+1}+p_{2 i}}{(\delta-1) q_{2 i+1}+q_{2 i}}$ \\
\hline
\end{tabular}

Proof. The following proof holds if $C_{i, j}$ has an even slope and both $i-1$ and $j+1$ are lower weakly exterior points. Other cases are deduced from Proposition 1 .

We bound the slopes obtained by extending $C_{i, j}$ to the front then extending $C_{i, j}$ to the back. Since $j+1$ is a lower weakly exterior point, $C_{i, j+1}$ has slope $z_{2 i+2}^{R}=\left[0, u_{1}, \ldots, u_{2 i}-1,1, \delta^{\prime}\right]$ (Proposition 11). Assuming that $C_{i, F(i)}$ has a slope that equals $\left[0, u_{1}, \ldots, u_{2 i}-1,1, \delta^{\prime}+\epsilon, u_{2 i+3}, \ldots, u_{p}\right]$ with $\epsilon$ being -1 or zero (from Proposition 1). Simple calculation brings: 


$$
\left.\left.\frac{1}{u_{2 i+3}+\frac{1}{\ldots+\frac{1}{u_{p}}}}=\epsilon^{\prime} \quad \text { with } \epsilon^{\prime} \in\right] 0,1\right]
$$

As a result, the slope of $C_{i, F(i)}$ equals $z^{R}\left(\epsilon_{R}\right)=\left[0, u_{1}, \ldots, u_{2 i}-1,1, \delta^{\prime}+\epsilon_{R}\right]$ with $\left.\left.\epsilon_{R} \in\right]-1,1\right]$. Eq. (2) and Eq. (3) still hold when partial coefficient are real values, we thus get

$$
z^{R}\left(\epsilon_{R}\right)=\frac{\left(\delta^{\prime}+\epsilon_{R}+1\right) p_{2 i}-p_{2 i-1}}{\left(\delta^{\prime}+\epsilon_{R}+1\right) q_{2 i}-q_{2 i-1}}
$$

We bound this slope for extremal values of $\epsilon_{R}$, giving

$$
\frac{\delta^{\prime} p_{2 i}-p_{2 i-1}}{\delta^{\prime} q_{2 i}-q_{2 i-1}} \leq z^{R}\left(\epsilon_{R}\right) \leq \frac{\left(\delta^{\prime}+2\right) p_{2 i}-p_{2 i-1}}{\left(\delta^{\prime}+2\right) q_{2 i}-q_{2 i-1}}
$$

Same reasoning applied to the back of the common part brings: $z_{2 i+2}^{L}=$ $\left[0, u_{1}, \ldots, u_{2 i}, \delta^{\prime}\right]$ and $z^{L}\left(\epsilon_{L}\right)=\left[0, u_{1}, \ldots, u_{2 i}, \delta^{\prime}+\epsilon_{L}\right]$ with $\left.\left.\epsilon_{L} \in\right]-1,1\right]$. Bounds are:

$$
\frac{\left(\delta^{\prime}+1\right) p_{2 i}+p_{2 i-1}}{\left(\delta^{\prime}+1\right) q_{2 i}+q_{2 i-1}} \leq z^{L}\left(\epsilon_{L}\right) \leq \frac{\left(\delta^{\prime}-1\right) p_{2 i}+p_{2 i-1}}{\left(\delta^{\prime}-1\right) q_{2 i}+q_{2 i-1}} .
$$

Furthermore, Theorem 1 give bounds on the slope difference $\Delta z$ of two consecutive maximal segments as a function of the parameters of their common part. For instance the case of an even slope with lower weakly exterior points give the tight bound

$$
\frac{2 \delta^{\prime}+3}{\left(\delta^{\prime 2}+3 \delta^{\prime}+2\right) q_{2 i}^{2}+q_{2 i} q_{2 i-1}-q_{2 i-1}^{2}}<|\Delta z|<\frac{2 \delta^{\prime}-1}{\left(\delta^{\prime 2}-\delta^{\prime}\right) q_{2 i}^{2}+q_{2 i} q_{2 i-1}-q_{2 i-1}^{2}} .
$$

We give below a coarser bound for $\Delta z$, but which is expressed only in terms of the slope denominator and the number of reversed-patterns:

$$
\frac{2 \delta^{\prime}+3}{\left(\delta^{\prime 2}+3 \delta^{\prime}+3\right) q_{2 i}^{2}}<|\Delta z|<\frac{2 \delta^{\prime}-1}{\left(\delta^{\prime 2}-\delta^{\prime}\right) q_{2 i}^{2}+\frac{1}{2} q_{2 i}+\frac{1}{2}} .
$$

In the other cases, similar formula are obtained. These formulas induce that the average slope difference between consecutive maximal segments could be determined, provided the average behaviour of $\delta, \delta^{\prime}$ and $q_{n}$ is known.

\section{Conclusion}

We have revisited a classical arithmetically-based DSS recognition algorithm with new parameters related to a combinatoric representation of DSS. New analytic relations have been established and the relation with the Stern-Brocot tree has been made explicit. At last, we have shown new geometric relations on maximal segments. The new parameters introduced in this paper seem to be good candidates to describe DSS and obtain new properties. It would be interesting 
to investigate the average asymptotic behavior of these parameters, that is $\delta$, $\delta^{\prime}$ and $q_{n}$, as functional of the grid step. This would lead us to estimate the asymptotic angle difference between consecutive maximal segments, a quantity related to curvature, and therefore to address the problem of finding a multigrid convergent curvature estimator. This study is thus a first step in this direction.

\section{References}

1. Debled-Renesson, I., Reveillès, J.P.: A linear algorithm for segmentation of discrete curves. Int. J. Pattern Recognit. Artif. Intell. 9 (1995) 635-662

2. Klette, R., Rosenfeld, A.: Digital straightness: a review. Discrete Appl. Math. 139(1-3) (2004) 197-230

3. Dorst, L., Smeulders, A.W.M.: Discrete representation of straight lines. IEEE Trans. Pattern Anal. Mach. Intell. 6 (1984) 450-463

4. Coeurjolly, D.: Supercover model and digital straight line recognition on irregular isothetic grids. In Andrès, E., Damiand, G., Lienhardt, P., eds.: Proc. DGCI'2005. Volume 3429 of LNCS., Springer (2005) 311-322

5. Berstel, J., De Luca, A.: Sturmian words, lyndon words and trees. Theoret. Comput. Sci. 178(1-2) (1997) 171-203

6. Voss, K.: Discrete Images, Objects, and Functions in $\mathbb{Z}^{n}$. Springer-Verlag (1993)

7. de Vieilleville, F., Lachaud, J.O., Feschet, F.: Maximal digital straight segments and convergence of discrete geometric estimators. In Kalviainen, H., Parkkinen, J., Kaarna, A., eds.: 14th Scandinavian Conference on Image Analysis. Number 3540 in LNCS, Springer-Verlag (2005) 988-997

8. F. de Vieilleville, J.O.L., Feschet, F.: Maximal digital straight segments and convergence of discrete geometric estimators. Research Report 1350-05, LaBRI, University Bordeaux 1, Talence, France (2005)

9. Feschet, F., Tougne, L.: Optimal time computation of the tangent of a discrete curve: application to the curvature. In: Proc. DGCI'1999. Number 1568 in LNCS, Springer Verlag (1999) 31-40

10. Klette, R., Rosenfeld, A.: Digital Geometry - Geometric Methods for Digital Picture Analysis. Morgan Kaufmann, San Francisco (2004)

11. Debled-Rennesson, I.: Etude et reconnaissance des droites et plans discrets. PhD thesis, Université Louis Pasteur, Strasbourg, France (1995)

12. Feschet., F., Tougne, L.: On the min DSS problem of closed discrete curves. In Lungo, A.D., Gesù, V.D., Kuba, A., eds.: IWCIA. Volume 12 of Electonic Notes in Discrete Math., Elsevier (2003)

13. Lachaud, J.O., Vialard, A., de Vieilleville, F.: Analysis and comparative evaluation of discrete tangent estimators. In Andrès, E., Damiand, G., Lienhardt, P., eds.: Proc. DGCI2005. LNCS 3429, Springer-Verlag (2005) 240-251

14. Reiter-Doerksen, H., Debled-Rennesson, I.: Convex and concave parts of digital curves. In: Dagstuhl Seminar "Geometric Properties from Incomplete Data". (2004)

15. Reveillès, J.P.: Géométrie discrète, calcul en nombres entiers et algorithmique. Thèse d'etat, Université Louis Pasteur, Strasbourg (1991)

16. Sivignon, I., Dupont, F., Chassery, J.M.: New results about digital intersections. In: Proc. DGCI'03. Volume 2886 of LNCS., Springer (2003) 102-113

17. Sivignon, I.: De la caractérisation des primitives à la reconstruction polyédrique de surfaces en géométrie discrète. $\mathrm{PhD}$ thesis, Institut national polytechnique de Grenoble, France (2004) 